5-1-2003

\title{
Steady State Analysis Of An M/D/2 Queue With Bernoulli Schedule Server Vacations
}

\author{
Kailash C. Madan \\ Yarmouk University, kailashmadan@hotmail.com \\ Walid Abu-Dayyeh \\ Yarmouk University \\ Firas Tayyan \\ Yarmouk University
}

Follow this and additional works at: http://digitalcommons.wayne.edu/jmasm

Part of the Applied Statistics Commons, Social and Behavioral Sciences Commons, and the Statistical Theory Commons

\section{Recommended Citation}

Madan, Kailash C.; Abu-Dayyeh, Walid; and Tayyan, Firas (2003) "Steady State Analysis Of An M/D/2 Queue With Bernoulli Schedule Server Vacations," Journal of Modern Applied Statistical Methods: Vol. 2 : Iss. 1 , Article 19.

DOI: $10.22237 /$ jmasm/1051748340

Available at: http://digitalcommons.wayne.edu/jmasm/vol2/iss1/19

This Regular Article is brought to you for free and open access by the Open Access Journals at DigitalCommons@WayneState. It has been accepted for inclusion in Journal of Modern Applied Statistical Methods by an authorized editor of DigitalCommons@WayneState. 


\title{
Steady State Analysis Of An M/D/2 Queue With Bernoulli Schedule Server Vacations
}

\author{
Kailash C. Madan Walid Abu-Dayyeh Firas Tayyan \\ Department of Statistics, Faculty of Science \\ Yarmouk University, Irbid, Jordan
}

We examine an M/D/2 queue with Bernoulli schedules and a single vacation policy. We have assumed Poisson arrivals waiting in a single queue and two parallel servers who provide identical deterministic service to customers on first-come, first-served basis. We consider two models; in one we assume that after completion of a service both servers can take a vacation while in the other we assume that only one may take a vacation. The vacation periods in both models are assumed to be exponential. We obtain steady state probability generating functions of system size for various states of the servers.

Key words: Two parallel servers, Bernoulli schedules, single vacation policy, deterministic service

Introduction

Vacation Queues have been studied by numerous researchers including Kleinrock (1983), Keilson and Servi (1986), Baba (1986), Doshi (1986,1990), Cramer (1989), Choi \& Park (1990), Borthakur \& Choudhury 1997), Madan (1992, 1999, 2001), to mention a few. Most of these authors have investigated single server queues assuming Bernoulli schedules or exhaustive service or generalized vacations among several other vacation policies with a single or multiple vacations. Madan and Saleh $(2001,2001,2001)$ have studied a single server queue with exponential service and deterministic vacations, deterministic service with exponential vacations and deterministic service with deterministic vacations, assuming Bernoulli schedules.

Those articles considered single server vacation models. Here, we study a queueing system with two parallel servers providing identical deterministic service assuming Bernoulli schedule server vacations with a single vacation policy.

Send correspondence to Kailash C. Madan, Department of Statistics, Faculty of Science, Yarmouk University, Irbid, Jordan. E-mail him at kailashmadan@hotmail.com.
We consider two models. In model A we assume that after a service completion both servers may take a vacation of identical exponential duration and in model $\mathrm{B}$, we assume that only one of the servers can take a vacation of exponential duration. In both models, we assume a single vacation policy which means that whenever a vacation period of a server ends, then he must join the system irrespective of whether there are customers waiting for service or not. That is, the server must join the system even if he finds the system empty on return. The following assumptions briefly describe our models:

Model A: Both Servers Can Take A Vacation. The Underlying Assumptions:

A - Customers arrive at the system one by one and their arrivals follow a Poisson distribution with mean arrival rate $\lambda,(\lambda>0)$.

B - Both servers provide identical deterministic (constant) service with constant service time of length $b,(b>0)$.

C - After every service, both servers together may take a vacation with probability $\mathrm{p}$ or continue to stay in the system with probability $1-p$. The vacation times follow an exponential distribution with mean vacation time $1 / \beta,(>0)$. 
D- All stochastic processes involved in the system are independent of each other.

\section{Definitions and Notations}

Define:

$B_{n}(t)$ : as the probability that at time $t$ both servers are available in the system providing service and there were $n(0)$ customers in the system when the current service started.

$N_{n}(t)$ : as the probability that at time $t$ there are $\mathrm{n}(0)$ customers in the system and both servers are on vacation.
$P_{n}(t)$ : as the probability that at time $t$ there are $\mathrm{n}(0)$ customers in the system without regardless of the state of the servers

$\mathrm{k}_{\mathrm{i}}$ : as the probability of $\mathrm{i}$ arrivals during a service period of constant length $b$.

Steady State Forward Equations of the System Assuming that the steady state exists, let $\lim _{t \rightarrow \infty} B_{n}(t)=B_{n}, \quad \lim _{t \rightarrow \infty} N_{n}(t)=N_{n}, \quad$ and $\lim _{t \rightarrow \infty} P_{n}(t)=P_{n}$. Thus, $B_{n}, N_{n}$ and $P_{n}$ denote the corresponding steady state probabilities. Then applying the usual probability reasoning we obtain the following set of steady state forward equations:

$$
\begin{aligned}
& B_{n}=(1-p) k_{n}\left[B_{0}+B_{1}+B_{2}\right]+(1-p) \sum_{i=3}^{n+2} B_{i} k_{n+2-i}+\beta N_{n}, \mathrm{n} \geq 0, \\
& (\lambda+\beta) N_{n}=\lambda N_{n-1}+p k_{n}\left[B_{0}+B_{1}+B_{2}\right]+p \sum_{i=3}^{n+2} B_{i} k_{n+2-i}, \mathrm{n} \geq 1, \\
& (\lambda+\beta) N_{0}=p k_{0}\left[B_{0}+B_{1}+B_{2}\right], \mathrm{n}=0 .
\end{aligned}
$$

Steady State Probability Generating Functions for the System Size

We define the following probability generation functions:

$$
\begin{aligned}
& B(z)=\sum_{n=0}^{\infty} B_{n} z^{n}, N(z)=\sum_{n=0}^{\infty} N_{n} z^{n}, P(z)=\sum_{n=0}^{\infty} P_{n} z^{n}, \\
& K(z)=\sum_{n=0}^{\infty} K_{n} z^{n}=\sum_{n=0}^{\infty} \frac{e^{-\lambda b}(\lambda b)^{n} z^{n}}{n !}=e^{-\lambda b} \sum_{n=0}^{\infty} \frac{(\lambda b z)^{n}}{n !}=e^{-\lambda b(1-z)},|z| \leq 1 .
\end{aligned}
$$

We multiply (1) by $z^{n+2}$ and add for all $\mathrm{n}=0,1,2 \ldots$ Then we have

$$
\begin{aligned}
& z^{2} \sum_{n=0}^{\infty} B_{n} z^{n}=(1-p) B_{0} z^{2} \sum_{n=0}^{\infty} k_{n} z^{n}+(1-p) B_{1} z^{2} \sum_{n=0}^{\infty} k_{n} z^{n}+(1-p) B_{2} z^{2} \sum_{n=0}^{\infty} k_{n} z^{n} \\
& +(1-p) \sum_{n=0}^{\infty} \sum_{i=3}^{n+2} B_{i} k_{n+2-i} z^{n+2}+\beta z^{2} \sum_{n=0}^{\infty} N_{n} z^{n}, \mathrm{n} \geq 0
\end{aligned}
$$


Then using (4) we obtain from (5)

$$
B(z)=\frac{\beta z^{2} N(z)+(1-p) B_{0}\left(z^{2}-1\right) e^{-\lambda b(1-z)}+(1-p) B_{1} z(z-1) e^{-\lambda b(1-z)}}{z^{2}-(1-p) e^{-\lambda b(1-z)}}
$$

Similarly we multiply (2) by $z^{n+2}$ and (3) by $z$ and add them for all $\mathrm{n}=0,1,2 \ldots$ Then we have

$$
\begin{gathered}
(\lambda+\beta) z^{2} \sum_{n=0}^{\infty} N_{n} z^{n}=\lambda z^{3} \sum_{n=0}^{\infty} N_{n} z^{n}+p B_{0} z^{2} \sum_{n=0}^{\infty} k_{n} z^{n}+p B_{1} z^{2} \sum_{n=0}^{\infty} k_{n} z^{n} \\
+p B_{2} z^{2} \sum_{n=0}^{\infty} k_{n} z^{n}+p \sum_{n=0}^{\infty} \sum_{i=3}^{n+2} B_{i} k_{n+2-i} z^{n+2}, \mathrm{n} \geq 0
\end{gathered}
$$

Using (4) we obtain from (7)

$$
N(z)=\frac{p B(z) e^{-\lambda b(1-z)}+p B_{0}\left(z^{2}-1\right) e^{-\lambda b(1-z)}+p B_{1} z(z-1) e^{-\lambda b(1-z)}}{(\lambda-\lambda z+\beta) z^{2}}
$$

Then we solve (6) and (8) simultaneously for $B(z)$ and $N(z)$ and obtain on simplifying

$$
\begin{aligned}
\mathrm{B}(\mathrm{z})= & {\left[\frac{\left[(1-p)\left(z^{2}-1\right) B_{0} e^{-\lambda b(1-z)}+(1-p) z(z-1) B_{1} e^{-\lambda b(1-z)}\right]\left[(\lambda-\lambda z+\beta) z^{2}\right]}{\left[z^{2}-(1-p) e^{-\lambda b(1-z)}\right]\left[(\lambda-\lambda z+\beta) z^{2}\right]-p \beta z^{2} e^{-\lambda b(1-z)}}\right.} \\
& +\frac{\beta z^{2}\left[p\left(z^{2}-1\right) B_{0} e^{-\lambda b(1-z)}+p z(z-1) B_{1} e^{-\lambda b(1-z)}\right]}{\left.\left[z^{2}-(1-p) e^{-\lambda b(1-z)}\right]\left[(\lambda-\lambda z+\beta) z^{2}\right]-p \beta z^{2} e^{-\lambda b(1-z)}\right]}, \\
\mathrm{N}(\mathrm{z})= & {\left[\frac{\left[p\left(z^{2}-1\right) B_{0} e^{-\lambda b(1-z)}+p z(z-1) B_{1} e^{-\lambda b(1-z)}\right]\left[z^{2}-(1-p) e^{-\lambda b(1-z)}\right]}{\left[z^{2}-(1-p) e^{-\lambda b(1-z)}\right]\left[(\lambda-\lambda z+\beta) z^{2}\right]-p \beta z^{2} e^{-\lambda b(1-z)}}\right.} \\
& \left.+\frac{p e^{-\lambda b(1-z)}\left[(1-p)\left(z^{2}-1\right) B_{0} e^{-\lambda b(1-z)}+(1-p) z(z-1) B_{1} e^{-\lambda b(1-z)}\right]}{\left[z^{2}-(1-p) e^{-\lambda b(1-z)}\right]\left[(\lambda-\lambda z+\beta) z^{2}\right]-p \beta z^{2} e^{-\lambda b(1-z)}}\right] .
\end{aligned}
$$

Hence, adding (9) and (10) we have

$$
P(z)=B(z)+N(z)
$$

Now we have to determine the unknown probabilities $B_{0}$ and $B_{1}$ which appear in the numerators of the right hand sides of equations (9), (10) and (11). For this purpose we use Rouche's theorem as follows. Let

$$
\begin{gathered}
f(z)=\left[z^{2}-(1-p) e^{-\lambda b(1-z)}\right]\left[(\lambda-\lambda z+\beta) z^{2}\right], \\
g(z)=-p \beta z^{2} e^{-\lambda b(1-z)} .
\end{gathered}
$$


Note that both $\mathrm{f}(\mathrm{z})$ and $\mathrm{g}(\mathrm{z})$ are regular on and inside $|\mathrm{z}|=1$. We aim to prove that $|f(z)| \geq|g(z)|$ on $|\mathrm{z}|=1$. Now, on $|\mathrm{z}|=1$,

$$
\begin{aligned}
& |f(z)|=\left|\left[z^{2}-(1-p) e^{-\lambda b(1-z)}\right]\left[(\lambda-\lambda z+\beta) z^{2}\right]\right| \\
& =\left|z^{2}-(1-p) e^{-\lambda b(1-z)}\right|\left|(\lambda-\lambda z+\beta) z^{2}\right| \\
& \geq\left[\left|z^{2}\right|-\left|(1-\mathrm{p}) \mathrm{e}^{-\lambda \mathrm{b}(1-z)}\right|\right]\left[|(\lambda-\lambda z+\beta)|\left|\mathrm{z}^{2}\right|\right] \\
& \left.=\mid 1-(1-p) e^{-\lambda b(1-z)}\right][\lambda-\lambda+\beta]=p \beta e^{-\lambda b(1-z)}=|g(z)| .
\end{aligned}
$$

Because $|f(z)| \geq|g(z)|$, therefore by Rouche's theorem $f(z)+g(z)$ has the same of zeros inside or on $|z|=1$ as that of $f(z)$. Now, it is easy to show that $f(z)$ has four zeros on or inside $|z|=1$. Therefore, $f(z)+g(z)$, i.e., the denominator of the right hand side of (11) has four zeros on or inside $|z|=1$. For each of these four zeros the numerator of the right hand side of (11) must vanish, thus giving us four linear equations in the two unknowns $B_{0}$ and $B_{1}$. Then two of these four equations are sufficient to determine the two unknowns, whereas the other two may just be redundant. Hence, the probability generating functions B (z), N (z) and P (z) obtained in (9), (10) and (11) can be completely determined.

Next, we shall use normalizing condition

$$
P(1)=B(1)+N(1)=1 .
$$

At $\mathrm{z}=1, P(1)=\frac{\text { zero }}{\text { zero }}$, therefore using L'Hopital's rule we have from (11)

$$
P(1)=\lim _{z \rightarrow 1} P(z)=\frac{(p+\beta)\left(2 B_{0}+B_{1}\right)}{2 \beta-\lambda b \beta-p \lambda}=1,
$$

which gives

$$
(p+\beta)\left(2 B_{0}+B_{1}\right)=2 \beta-\lambda b \beta-p \lambda .
$$

Equation (14) will hold only if $2 \beta-\lambda b \beta-p \lambda>0$ which gives the steady state condition

$$
\frac{\lambda(b \beta+p)}{2 \beta}<1 .
$$

Note that when there are no server vacations, then with $p=0, N_{n}=0$ for all $\mathrm{n} \geq 0$, equation (10) yields $N(z)=0$ as it should be. Further, equations (9) and (15) respectively give

$$
\begin{aligned}
& \mathrm{B}(\mathrm{z})=\left[\frac{\left[\left(z^{2}-1\right) B_{0} e^{-\lambda b(1-z)}+z(z-1) B_{1} e^{-\lambda b(1-z)}\right]}{\left[z^{2}-e^{-\lambda b(1-z)}\right]},\right. \\
& \lambda b<2 .
\end{aligned}
$$


Note that (16) and (17) are the known results for the M/D/c queue for $c=2$. (See Kashyap and Chaudhury, 1988, p. 60-61.)

\section{Model B: Only One Server At A Time Can Take A} Vacation

The Underlying Assumptions.

In this case, the assumptions (a), (b) and (d) in section 2.1 for the previous case are the same. However, assumption (c) is different in this case under which we assume that after every service completion, only one server may take a vacation with probability $p$ or continue to stay in the system with probability $1-p$. The vacation times follow an exponential distribution with mean vacation time $1 / \beta,(\beta>0)$.

\section{Definitions and Notations}

We define:

$B_{n}(t)$ : as the probability that at time $t$ both servers are available in the system providing service and there were $n(\geq 0)$ customers in the system when the current service started.

$O_{n}(t)$ : as the probability that at time t only one server is available in the system providing service and there were $n(\geq 0)$ customers when the current service started.

$P_{n}(t)$ : as the probability that at time $t$ there are $\mathrm{n}$ $(\geq 0)$ customers in the system regardless of the state of the servers.

Steady State Forward Equations of the System Assuming that steady state exists, we let $\lim _{t \rightarrow \infty} B_{n}(t)=B_{n}, \quad \lim _{t \rightarrow \infty} O_{n}(t)=O_{n} \quad$ and $\lim _{t \rightarrow \infty} P_{n}(t)=P_{n}$. Thus $B_{n}, O_{n}$ and $P_{n}$ denote the corresponding steady state probabilities. Then we obtain the following set of steady state equations:

$$
\begin{aligned}
& B_{n}=(1-p) k_{n}\left[B_{0}+B_{1}+B_{2}\right]+(1-p) \sum_{i=3}^{n+2} B_{i} k_{n+2-i}+\beta O_{n}, \mathrm{n} \geq 0, \\
& (1+\beta) O_{n}=O_{0} k_{n}+O_{1} k_{n}+\sum_{j=2}^{n+1} O_{j} k_{n+1-j}+p k_{n}\left[B_{0}+B_{1}+B_{2}\right]+p \sum_{i=3}^{n+2} B_{i} k_{n+2-i}, \mathrm{n} \geq 0 .
\end{aligned}
$$

Steady State Probability Generating Functions for the System Size

In addition to the probability generating functions defined in (4a) and $4 b$ ) in section 2.4 , we define the following probability generation function:

$$
O(z)=\sum_{n=0}^{\infty} O_{n} z^{n},|\mathrm{z}| \leq 1 .
$$

We multiply both sides of equation (16) by $z^{n+2}$ and add for all $n=0,1,2 \ldots$ Thus we have

$$
\begin{aligned}
& z^{2} \sum_{n=0}^{\infty} B_{n} z^{n}=(1-p) B_{0} z^{2} \sum_{n=0}^{\infty} k_{n} z^{n}+(1-p) B_{1} z^{2} \sum_{n=0}^{\infty} k_{n} z^{n}+(1-p) B_{2} z^{2} \sum_{n=0}^{\infty} k_{n} z^{n} \\
& +(1-p) \sum_{n=0}^{\infty} \sum_{i=3}^{n+2} B_{i} k_{n+2-i} z^{n+2}+\beta z^{2} \sum_{n=0}^{\infty} O_{n} z^{n}, \mathrm{n} \geq 0
\end{aligned}
$$

Then using (4a), (4b) and (20) we obtain from (21) 


$$
B(z)=\frac{\beta z^{2} O(z)+(1-p) B_{0}\left(z^{2}-1\right) e^{-\lambda b(1-z)}+(1-p) B_{1} z(z-1) e^{-\lambda b(1-z)}}{z^{2}-(1-p) e^{-\lambda b(1-z)}} .
$$

Similarly, we multiply both sides of (19) by $z^{n+2}$ and add them for all $\mathrm{n}=0,1,2 \ldots$ Then we have

$$
\begin{gathered}
(1+\beta) z^{2} \sum_{n=0}^{\infty} O_{n} z^{n}=O_{0} z^{2} \sum_{n=0}^{\infty} k_{n} z^{n}+O_{1} z^{2} \sum_{n=0}^{\infty} k_{n} z^{n}+\sum_{n=0}^{\infty} \sum_{j=2}^{n+1} O_{j} k_{n+1-j} z^{n+2} \\
+p B_{0} z^{2} \sum_{n=0}^{\infty} k_{n} z^{n}+p B_{1} z^{2} \sum_{n=0}^{\infty} k_{n} z^{n}+p B_{2} z^{2} \sum_{n=0}^{\infty} k_{n} z^{n} \\
+p \sum_{n=0}^{\infty} \sum_{i=3}^{n+2} B_{i} k_{n+2-i} z^{n+2}, \mathrm{n} \geq 0
\end{gathered}
$$

Then using (4a), (4b) and (18) we obtain from (23)

$$
O(z)=\frac{p B(z) e^{-\lambda b(1-z)}+p B_{0}\left(z^{2}-1\right) e^{-\lambda b(1-z)}+p B_{1} z(z-1) e^{-\lambda b(1-z)}+z(z-1) O_{0} e^{-\lambda b(1-z)}}{(1+\beta) z^{2}-z e^{-\lambda b(1-z)}} .
$$

Then, we solve equations (22) and (24) simultaneously for $B(z)$ and $O(z)$ and obtain

$$
\begin{array}{r}
\mathrm{B}(\mathrm{z})=\left[\frac{\left[(1-p)\left(z^{2}-1\right) B_{0} e^{-\lambda b(1-z)}+(1-p) z(z-1) B_{1} e^{-\lambda b(1-z)}\right]\left[(1+\beta) z^{2}-z e^{-\lambda b(1-z)}\right]}{\left[z^{2}-(1-p) e^{-\lambda b(1-z)}\right]\left[(1+\beta) z^{2}-z e^{-\lambda b(1-z)}\right]-p \beta z^{2} e^{-\lambda b(1-z)}}\right. \\
+\frac{\beta z^{2}\left[p\left(z^{2}-1\right) B_{0} e^{-\lambda b(1-z)}+p z(z-1) B_{1} e^{-\lambda b(1-z)}+z(z-1) O_{n} e^{-\lambda b(1-z)}\right]}{\left.\left[z^{2}-(1-p) e^{-\lambda b(1-z)}\right]\left[(1+\beta) z^{2}-z e^{-\lambda b(1-z)}\right]-p \beta z^{2} e^{-\lambda b(1-z)}\right],} \\
\mathrm{O}(\mathrm{z})=\left[\frac{\left[p\left(z^{2}-1\right) B_{0} e^{-\lambda b(1-z)}+p z(z-1) B_{1} e^{-\lambda b(1-z)}+z(z-1) O_{0} e^{-\lambda b(1-z)}\right]\left[z^{2}-(1-p) e^{-\lambda b(1-z)}\right]}{\left[z^{2}-(1-p) e^{-\lambda b(1-z)}\right]\left[(1+\beta) z^{2}-z e^{-\lambda b(1-z)}\right]-p \beta z^{2} e^{-\lambda b(1-z)}}\right. \\
+\frac{p e^{-\lambda b(1-z)}\left[(1-p)\left(z^{2}-1\right) B_{0} e^{-\lambda b(1-z)}+(1-p) z(z-1) B_{1} e^{-\lambda b(1-z)}\right]}{\left.\left[z^{2}-(1-p) e^{-\lambda b(1-z)}\right]\left[(1+\beta) z^{2}-z e^{-\lambda b(1-z)}\right]-p \beta z^{2} e^{-\lambda b(1-z)}\right]}
\end{array}
$$

Then adding (25) and (26), we obtain

$$
P(z)=B(z)+O(z)
$$

The unknowns $B_{0}, B_{1}$ and $O_{0}$ can be determined by applying Rouche's theorem as before. Hence, the probability generating functions $\mathrm{B}(\mathrm{z}), \mathrm{O}(\mathrm{z})$ and $\mathrm{P}(\mathrm{z})$ can be completely determined. 
Further, we use the normalizing condition

$$
P(1)=B(1)+O(1)=1
$$

At $\mathrm{z}=1$, because $P(1)=\frac{\text { zero }}{\text { zero }}$, and hence, using L'Hopital's rule we have from

$$
P(1)=\lim _{z \rightarrow 1} P(z)=\frac{(p+\beta)\left(2 B_{0}+B_{1}+O_{0}\right)}{2 \beta-\lambda b \beta+p-p \lambda b}=1
$$

which gives

$$
(p+\beta)\left(2 B_{0}+B_{1}+O_{0}\right)=2 \beta-\lambda b \beta+p-p \lambda b
$$

Equation (30) will hold only if $2 \beta-\lambda b \beta+p-p \lambda b>0$ which yields the steady state condition

$$
\frac{\lambda b(\beta+p)}{2 \beta+p}<1
$$

Again note that when there are no server vacations, then with $p=0$ and $O_{n}=0$ for all $\mathrm{n} \geq 0$, equation (26) yields $\mathrm{O}(\mathrm{z})=0$ as it should be. Further, (25) and (31) respectively give

$$
\begin{aligned}
& \mathrm{B}(\mathrm{z})=\left[\frac{\left[\left(z^{2}-1\right) B_{0} e^{-\lambda b(1-z)}+z(z-1) B_{1} e^{-\lambda b(1-z)}\right]}{\left[z^{2}-e^{-\lambda b(1-z)}\right]}\right], \\
& \lambda b<2 .
\end{aligned}
$$

Note that (32) and (33) are the same known results for the M/D/c queue for $c=2$ as in section 2.4. (See Kashyap \& Chaudhury, 1988, p. 60-61.)

\section{References}

Baba, Y. (1986): On the queue with vacation time. Operations Research Letters, 5, 9398.

Borthakur, A. \& Choudhury, G. (1997). On a batch arrival Poisson queue with generalized vacation. Sankhya Ser. B., 59, 369-383.

Choi, B. D., \& Park, K.K. (1990): The $\mathrm{M} / \mathrm{G} / 1$ retrial queue with Bernoulli schedules, Queueing Systems, 7, 219-228.

Cramer, M. (1989). Stationary distribution in a queueing system with vacation times and limited service, Queueing Systems Theory and Applications, 4, 1, 57-68.
Doshi, B.T. (1986). Queueing systems with vacations - a survey. Queueing Systems, 1, 29-66.

Doshi, B.T. (1990). Conditional and Unconditional distributions for the M/G/1 queue with vacations. Questa, 7, 229-252.

Gross, C., \& Harris, C.M. (1985): The fundamentals of queueing theory. NY: John Wiley and Sons.

Kashyap, B. R. K., \& Chaudhry, M. L. (1988). An introduction to queueing theory. Ontario, Canada: A and A Publications. 
Keilson, J., \& Servi, L.D. (1986): Oscillating random walk models for $\mathrm{GI} / \mathrm{G} / 1$ vacation systems with Bernoulli schedules. Journal of Applied Probability, 23, 790-802.

Kleinrock, L. (1983). On the M/G/1 queue with rest periods and certain service independent queueing disciplines. Operations Research, 31(4), 705-719.

Madan, K. C. (1992). An M/G/1 queueing system with compulsory server vacations. Trabajos de Investigacion Operativa, 7(1), 105115.

Madan, K. C. (1999). An M/G/1 queue with optional deterministic server vacations. Metron, LVII, 3-4, 83-95.

Madan, K.C. (2001). On a single server queue with two-stage heterogeneous service and deterministic server vacations. Intern. J. Systems Science, 32(7), 837-844.
Madan, K. C., \& Saleh, M. F. (2001). On $\mathrm{M} / \mathrm{D} / 1$ queue with general server vacations. International Journal of Man. \& Inform. Sc., 12(2), 25-37.

Madan, K. C. \& Saleh, M. F. (2001). On Single server vacation with deterministic service or deterministic vacations. Calcuutta Statistical Association Bulletin, 51, 203-

204; 225-241.

Madan, K. C., \& Saleh, M. F. (2001). On $\mathrm{M} / \mathrm{D} / 1$ queue with deterministic vacations. Systems Science, 27(2), 107-118.

Medhi, J. (1982). Stochastic Processes. NY: Wiley Eastern. 